\title{
Hyperchaos, adaptive control and synchronization of a novel 4-D hyperchaotic system with two quadratic nonlinearities
}

\author{
SUNDARAPANDIAN VAIDYANATHAN
}

\begin{abstract}
This research work announces an eleven-term novel 4-D hyperchaotic system with two quadratic nonlinearities. We describe the qualitative properties of the novel 4-D hyperchaotic system and illustrate their phase portraits. We show that the novel 4-D hyperchaotic system has two unstable equilibrium points. The novel 4-D hyperchaotic system has the Lyapunov exponents $L_{1}=3.1575, L_{2}=0.3035, L_{3}=0$ and $L_{4}=-33.4180$. The Kaplan-Yorke dimension of this novel hyperchaotic system is found as $D_{K Y}=3.1026$. Since the sum of the Lyapunov exponents of the novel hyperchaotic system is negative, we deduce that the novel hyperchaotic system is dissipative. Next, an adaptive controller is designed to stabilize the novel 4-D hyperchaotic system with unknown system parameters. Moreover, an adaptive controller is designed to achieve global hyperchaos synchronization of the identical novel 4-D hyperchaotic systems with unknown system parameters. The adaptive control results are established using Lyapunov stability theory. MATLAB simulations are depicted to illustrate all the main results derived in this research work.
\end{abstract}

Key words: chaos, hyperchaos, control, synchronization, Lyapunov exponents.

\section{Introduction}

Chaos theory describes the qualitative study of unstable aperiodic behaviour in deterministic nonlinear dynamical systems. For the motion of a dynamical system to be chaotic, the system variables should contain nonlinear terms and it must satisfy three properties: boundedness, infinite recurrence and sensitive dependence on initial conditions [1,2].

The Lyapunov exponent of a dynamical system is a quantity that characterizes the rate of separation of infinitesimally close trajectories. The sensitive dependence on initial conditions of a dynamical system is characterized by the presence of a positive Lyapunov exponent. A positive Lyapunov exponent reflects a direction of stretching and folding and along with phase-space compactness indicates the presence of chaos in a dynamical

The author is with Research and Development Centre, Vel Tech University, Avadi, Chennai- 600062, Tamil Nadu, India. E-mail: sundarcontrol@gmail.com

Received 9.05.2016. 
system. An $n$-dimensional dynamical system has a spectrum of $n$ Lyapunov exponents and the maximal Lyapunov exponent (MLE) of a chaotic system is defined as the largest positive Lyapunov exponent of the system.

Chaos has developed over time. For example, Ruelle and Takens [3] proposed a theory for the onset of turbulence in fluids, based on abstract considerations about strange attractors. Later, May [4] found examples of chaos in iterated mappings arising in population biology. Feigenbaum [5] discovered that there are certain universal laws governing the transition from regular to chaotic behaviours. That is, completely different systems can go chaotic in the same way, thus, linking chaos and phase transitions.

The first famous chaotic system was accidentally discovered by Lorenz, when he was designing a 3-D model for atmospheric convection in 1963 [6]. Subsequently, Rössler discovered a 3-D chaotic system in 1976 [7], which is algebraically simpler than the Lorenz system. Indeed, Lorenz's system is a seven-term chaotic system with two quadratic nonlinearities, while Rössler's system is a seven-term chaotic system with just one quadratic nonlinearity.

Some well-known paradigms of 3-D chaotic systems are Arneodo system [8], Sprott systems [9], Chen system [10], Lü-Chen system [11], Liu system [12], Cai system [13], T-system [14], etc. Many new chaotic systems have been also discovered like Li system [15], Sundarapandian systems [16, 17], Vaidyanathan systems [18, 19, 20, 21, 22, 23, 24], Pehlivan system [25], Akgul system [26], Jafari system [27], Pham system [28, 29, 30, 31], Tacha system [32], etc.

Chaos theory has applications in several fields of science and engineering such as oscillators [33, 34], chemical reactions [35, 36], biology [37, 38], ecology [39, 40], neural networks [41, 42], gyros [43], Tokamak system [44, 45],neurology [46, 47, 48], circuits $[49,50]$, etc.

A hyperchaotic system is generally defined as a chaotic system with at least two positive Lyapunov exponents [1,2]. Thus, the hyperchaotic systems have more complex dynamical behaviour and hence they have miscellaneous applications in engineering $[1,2]$.

The minimum dimension for an autonomous, continuous-time, hyperchaotic system is four. Since the discovery of a first 4-D hyperchaotic system by Rössler in 1979 [65], many 4-D hyperchaotic systems have been found in the literature such as hyperchaotic Lorenz system [66], hyperchaotic Lü system [67], hyperchaotic Chen system [68], hyperchaotic Wang system [69], hyperchaotic Newton-Leipnik system [70], hyperchaotic Vaidyanathan system $[71,72]$, etc.

The study of control of a chaotic system investigates methods for designing feedback control laws that globally or locally asymptotically stabilize or regulate the outputs of a chaotic system [73].

Chaos synchronization problem deals with the synchronization of a couple of systems called the master or drive system and the slave or response system. To solve this problem, control laws are designed so that the output of the slave system tracks the output of the master system asymptotically with time [73, 74]. 
Because of the butterfly effect, the synchronization of chaotic systems is a challenging problem in the chaos literature even when the initial conditions of the master and slave systems are nearly identical because of the exponential divergence of the outputs of the two systems in the absence of any control.

This research work announces an eleven-term novel 4-D hyperchaotic system with two quadratic nonlinearities. We describe the qualitative properties of the novel 4-D hyperchaotic system and illustrate their phase portraits. We show that the novel 4-D hyperchaotic system has two unstable equilibrium points.

We also show that the novel 4-D hyperchaotic system has the Lyapunov exponents $L_{1}=3.1575, L_{2}=0.3035, L_{3}=0$ and $L_{4}=-33.4180$. The Kaplan-Yorke dimension of this novel hyperchaotic system is found as $D_{K Y}=3.1026$. Since the sum of the Lyapunov exponents of the novel hyperchaotic system is negative, we deduce that the novel hyperchaotic system is dissipative.

Next, an adaptive controller is designed to stabilize the novel 4-D hyperchaotic system with unknown system parameters. Moreover, an adaptive controller is designed to achieve global hyperchaos synchronization of the identical novel 4-D hyperchaotic systems with unknown system parameters. The adaptive control results are established using Lyapunov stability theory [75]. MATLAB simulations are depicted to illustrate all the main results derived in this research work.

\section{A novel 4-D hyperchaotic system}

In this work, we propose a novel 4-D hyperchaotic system given by

$$
\left\{\begin{array}{l}
\dot{x}_{1}=a\left(x_{2}-x_{1}\right)+x_{4} \\
\dot{x}_{2}=b x_{1}-x_{2}-x_{1} x_{3} \\
\dot{x}_{3}=-x_{1}-c x_{3}+x_{1} x_{2}+x_{4} \\
\dot{x}_{4}=-p x_{2}
\end{array}\right.
$$

In (1), $x_{1}, x_{2}, x_{3}, x_{4}$ are the states and $a, b, c, p$ are positive, constant, parameters.

In this work, we show that the 4-D system (1) is hyperchaotic when the parameter values are taken as

$$
a=24, b=125, c=5, p=10
$$

Also, for these parameter values, the Lyapunov exponents of the novel 4-D system (1) are calculated as

$$
L_{1}=3.1575, L_{2}=0.3035, L_{3}=0, L_{4}=-33.4180
$$

Since there are two positive Lyapunov exponents in (3), it is immediate that the proposed novel 4-D system (1) is hyperchaotic. 
Also, the maximal Lyapunov exponent (MLE) of the system (1) is obtained as $L_{1}=3.1575$, which is a large number. This shows the high complexity of the novel 4-D hyperchaotic system (1).

The system (1) is dissipative, because

$$
L_{1}+L_{2}+L_{3}+L_{4}=-29.9570<0
$$

Also, the Kaplan-Yorke dimension of the 4-D hyperchaotic system (1) is found as

$$
D_{K Y}=3+\frac{L_{1}+L_{2}+L_{3}}{\left|L_{4}\right|}=3.1036
$$

which is fractional. Thus, the 4-D hyperchaotic system (1) has a strange attractor of fractional Kaplan-Yorke dimension.

For numerical simulations, we take the initial state of the hyperchaotic system (1) as

$$
x_{1}(0)=0.2, \quad x_{2}(0)=0.2, \quad x_{3}(0)=0.2, x_{4}(0)=0.2
$$

Figs. 1-4 depict the 3-D phase portraits of the novel 4-D hyperchaotic system (1) in $\left(x_{1}, x_{2}, x_{3}\right),\left(x_{1}, x_{2}, x_{4}\right),\left(x_{1}, x_{3}, x_{4}\right)$ and $\left(x_{2}, x_{3}, x_{4}\right)$ spaces, respectively. From these figures, it is clear the novel 4-D hyperchaotic system (1) exhibits a two-wing attractor.

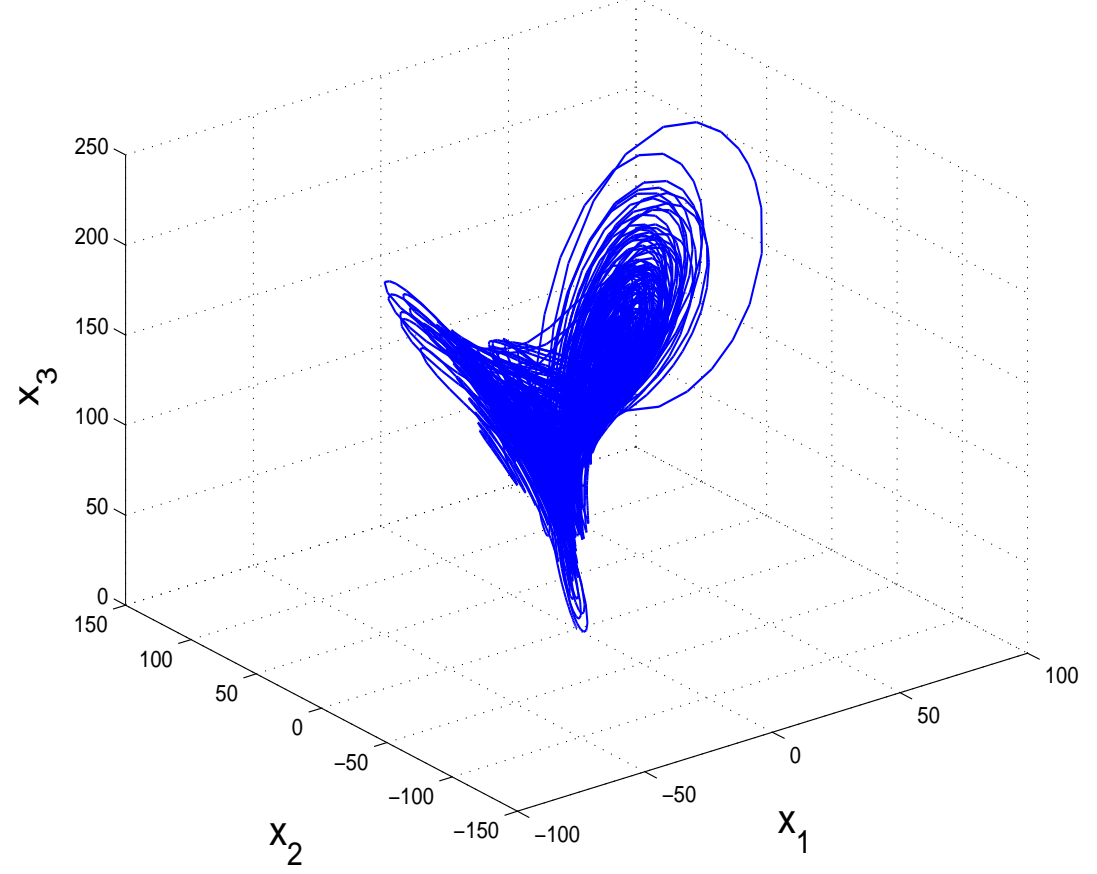

Figure 1: 3-D projection of the novel hyperchaotic system on $\left(x_{1}, x_{2}, x_{3}\right)$ space 


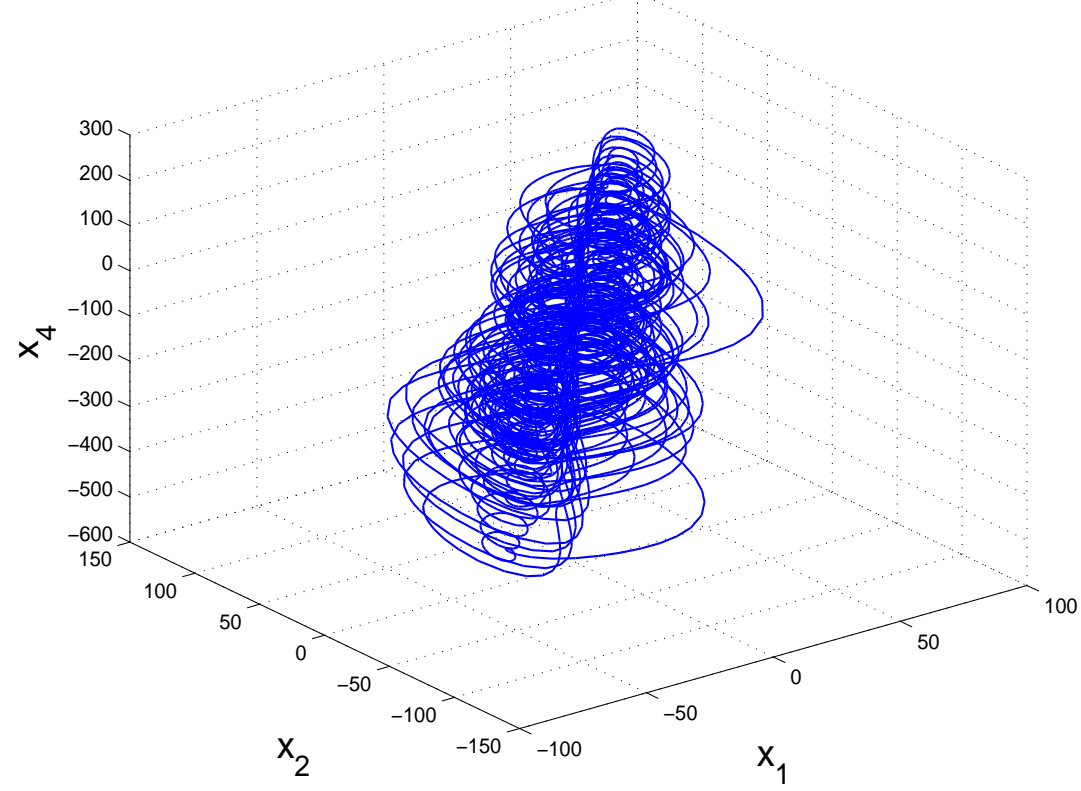

Figure 2: 3-D projection of the novel hyperchaotic system on $\left(x_{1}, x_{2}, x_{4}\right)$ space

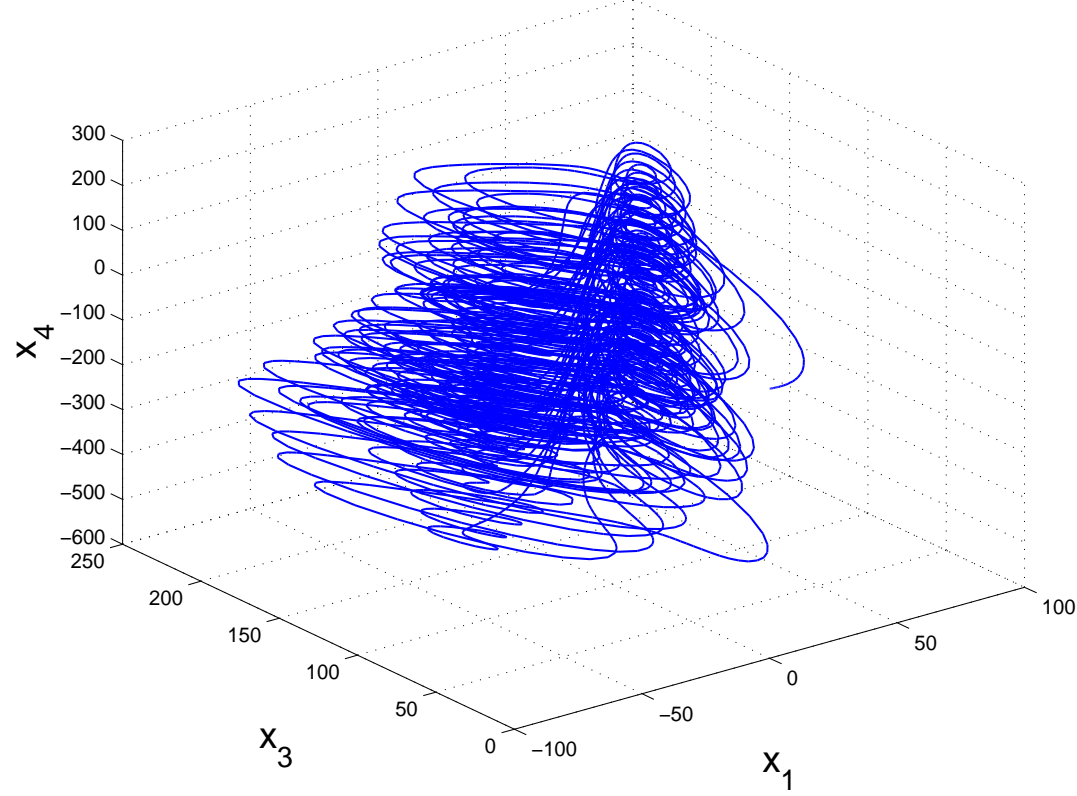

Figure 3: 3-D projection of the novel hyperchaotic system on $\left(x_{1}, x_{3}, x_{4}\right)$ space 


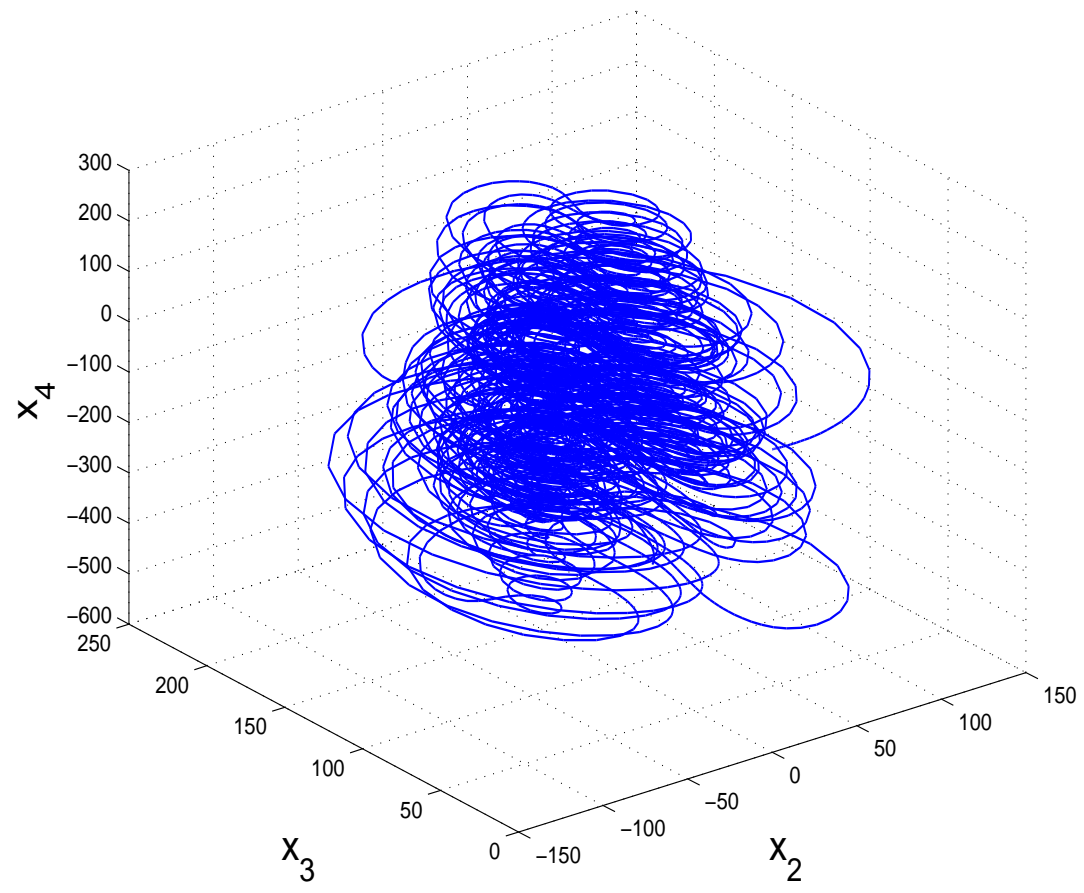

Figure 4: 3-D projection of the novel hyperchaotic system on $\left(x_{2}, x_{3}, x_{4}\right)$ space

\section{Analysis of the novel hyperchaotic system}

\subsection{Dissipativity}

In vector notation, the novel 4-D hyperchaotic system (1) can be expressed as

$$
\dot{\boldsymbol{x}}=f(\boldsymbol{x})=\left[\begin{array}{l}
f_{1}\left(x_{1}, x_{2}, x_{3}, x_{4}\right) \\
f_{2}\left(x_{1}, x_{2}, x_{3}, x_{4}\right) \\
f_{3}\left(x_{1}, x_{2}, x_{3}, x_{4}\right) \\
f_{4}\left(x_{1}, x_{2}, x_{3}, x_{4}\right)
\end{array}\right],
$$

where

$$
\left\{\begin{array}{l}
f_{1}\left(x_{1}, x_{2}, x_{3}, x_{4}\right)=a\left(x_{2}-x_{1}\right)+x_{4} \\
f_{2}\left(x_{1}, x_{2}, x_{3}, x_{4}\right)=b x_{1}-x_{2}-x_{1} x_{3} \\
f_{3}\left(x_{1}, x_{2}, x_{3}, x_{4}\right)=-x_{1}-c x_{3}+x_{1} x_{2}+x_{4} \\
f_{4}\left(x_{1}, x_{2}, x_{3}, x_{4}\right)=-p x_{2}
\end{array}\right.
$$

Let $\Omega$ be any region in $\Re^{4}$ with a smooth boundary and also, $\Omega(t)=\Phi_{t}(\Omega)$, where $\Phi_{t}$ is the flow of $f$. Furthermore, let $V(t)$ denote the volume of $\Omega(t)$. 
By Liouville's theorem, we know that

$$
\dot{V}(t)=\int_{\Omega(t)}(\nabla \cdot f) d x_{1} d x_{2} d x_{3} d x_{4}
$$

The divergence of the novel 4-D system (1) is found as:

$$
\nabla \cdot f=\frac{\partial f_{1}}{\partial x_{1}}+\frac{\partial f_{2}}{\partial x_{2}}+\frac{\partial f_{3}}{\partial x_{3}}+\frac{\partial f_{1}}{\partial x_{4}}=-a-1-c=-\mu
$$

where $\mu$ is defined as

$$
\mu=a+1+c
$$

For the choice of parameter values given in (2), we find that $\mu=30>0$.

Inserting the value of $\nabla \cdot f$ from (10) into (9), we get

$$
\dot{V}(t)=\int_{\Omega(t)}(-\mu) d x_{1} d x_{2} d x_{3} d x_{4}=-\mu V(t)
$$

Integrating the first order linear differential equation (12), we get

$$
V(t)=\exp (-\mu t) V(0)
$$

Since $\mu>0$, it follows from (13) that $V(t) \rightarrow 0$ exponentially as $t \rightarrow \infty$. This shows that the novel 4-D hyperchaotic system (1) is dissipative. Hence, the system limit sets are ultimately confined into a specific limit set of zero volume, and the asymptotic motion of the novel 4-D hyperchaotic system (1) settles onto a strange attractor of the system.

\subsection{Equilibrium Points}

The equilibrium points of the novel 4-D hyperchaotic system (1) are obtained by solving the equations

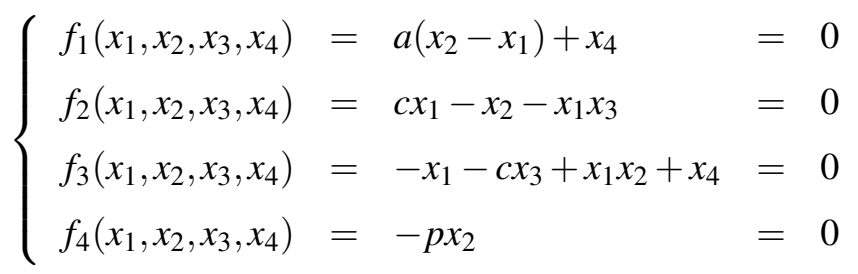

We take the parameter values as in the equation (2).

Solving the system (14), we obtain two equilibrium points of the system (1) given by

$$
E_{0}=\left[\begin{array}{l}
0 \\
0 \\
0 \\
0
\end{array}\right], \quad E_{1}=\left[\begin{array}{c}
27.1739 \\
0.0000 \\
125.0000 \\
652.1739
\end{array}\right]
$$


The Jacobian matrix of the 4-D hyperchaotic system (1) at any point $\boldsymbol{x} \in \mathfrak{R}^{4}$ is given by

$$
J(\boldsymbol{x})=\left[\begin{array}{rrrr}
-a & a & 0 & 1 \\
b-x_{3} & -1 & -x_{1} & 0 \\
-1+x_{2} & x_{1} & -c & 1 \\
0 & -p & 0 & 0
\end{array}\right]=\left[\begin{array}{rrrr}
-24 & 24 & 0 & 1 \\
125-x_{3} & -1 & -x_{1} & 0 \\
-1+x_{2} & x_{1} & -5 & 1 \\
0 & -10 & 0 & 0
\end{array}\right]
$$

The Jacobian matrix of the system (1) at $E_{0}$ is found as

$$
J_{0}=J\left(E_{0}\right)=\left[\begin{array}{rrrr}
-24 & 24 & 0 & 1 \\
125 & -1 & 0 & 0 \\
-1 & 0 & -5 & 1 \\
0 & -10 & 0 & 0
\end{array}\right]
$$

The eigenvalues of the matrix $J_{0}$ are numerically obtained as

$$
\lambda_{1}=-5, \lambda_{2}=-68.6290, \lambda_{3}=0.4215, \lambda_{4}=43.2074
$$

Thus, the equilibrium point $E_{0}$ is a saddle-point, which is unstable.

Next, the Jacobian matrix of the system (1) at $E_{1}$ is found as

$$
J_{1}=J\left(E_{1}\right)=\left[\begin{array}{rrrr}
-24 & 24 & 0 & 1 \\
125 & -1 & 0 & 0 \\
-1 & 0 & -5 & 1 \\
0 & -10 & 0 & 0
\end{array}\right]
$$

The eigenvalues of the matrix $J_{1}$ are numerically obtained as

$$
\lambda_{1}=0.3624, \lambda_{2}=-23.4294, \lambda_{3,4}=-3.4665 \pm 26.9067 i
$$

Thus, the equilibrium point $E_{1}$ is a saddle-focus, which is also unstable.

\subsection{Symmetry}

It is easy to see that the novel 4-D hyperchaotic system (1) is invariant under the coordinates transformation

$$
\left(x_{1}, x_{2}, x_{3}, x_{4}\right) \mapsto\left(-x_{1},-x_{2}, x_{3},-x_{4}\right)
$$

which shows that the novel system (1) has rotation symmetry about the $x_{3}$ axis. As a consequence, it follows that any non-trivial trajectory of the system (1) must have a twin trajectory. 


\subsection{Invariance}

It is easy to see that the $x_{3}$-axis is invariant under the flow of the novel 4-D hyperchaotic system (1). The invariant motion along the $x_{3}$-axis is characterized by the scalar dynamics

$$
\dot{x}_{3}=-c x_{3}, \quad(c>0)
$$

which is globally exponentially stable.

\subsection{Lyapunov exponents and Kaplan-Yorke dimension}

For the parameter values given in the equation (2), the Lyapunov exponents of the novel 4-D hyperchaotic system (1) are calculated as

$$
L_{1}=3.1575, L_{2}=0.3035, L_{3}=0, L_{4}=-33.4180
$$

Thus, the novel 4-D hyperchaotic system (1) has two positive Lyapunov exponents. Also, the maximal Lyapunov exponent (MLE) of the system (1)is obtained as $L_{1}=3.1575$, which is a large value. This shows the high complexity of the novel 4-D hyperchaotic system (1).

Also, the Kaplan-Yorke dimension of the novel hyperchaotic system (1) is obtained as

$$
D_{K Y}=3+\frac{L_{1}+L_{2}+L_{3}}{\left|L_{4}\right|}=3.1036
$$

which is fractional.

Since the novel 4-D hyperchaotic system (1) has two positive Lyapunov exponents, it has a very complex dynamics and the system trajectories can expand in two different directions.

\section{Adaptive control of the novel hyperchaotic system with unknown parameters}

In this section, we use adaptive control method to derive an adaptive feedback control law for globally stabilizing the novel 4-D hyperchaotic system with unknown parameters.

Thus, we consider the novel 4-D hyperchaotic system given by

$$
\left\{\begin{array}{l}
\dot{x}_{1}=a\left(x_{2}-x_{1}\right)+x_{4}+u_{1} \\
\dot{x}_{2}=b x_{1}-x_{2}-x_{1} x_{3}+u_{2} \\
\dot{x}_{3}=-x_{1}-c x_{3}+x_{1} x_{2}+x_{4}+u_{3} \\
\dot{x}_{4}=-p x_{2}+u_{4}
\end{array}\right.
$$

In (25), $x_{1}, x_{2}, x_{3}, x_{4}$ are the states and $u_{1}, u_{2}, u_{3}, u_{4}$ are the adaptive controls to be determined using estimates $\hat{a}(t), \hat{b}(t), \hat{c}(t), \hat{p}(t)$ for the unknown parameters $a, b, c, p$, respectively. 
We consider the adaptive feedback control law

$$
\left\{\begin{array}{l}
u_{1}=-\hat{a}(t)\left(x_{2}-x_{1}\right)-x_{4}-k_{1} x_{1} \\
u_{2}=-\hat{b}(t) x_{1}+x_{2}+x_{1} x_{3}-k_{2} x_{2} \\
u_{3}=x_{1}+\hat{c}(t) x_{3}-x_{1} x_{2}-x_{4}-k_{3} x_{3} \\
u_{4}=\hat{p}(t) x_{2}-k_{4} x_{4}
\end{array}\right.
$$

where $k_{1}, k_{2}, k_{3}, k_{4}$ are positive gain constants.

Substituting (26) into (25), we get the closed-loop plant dynamics as

$$
\left\{\begin{aligned}
\dot{x}_{1} & =[a-\hat{a}(t)]\left(x_{2}-x_{1}\right)-k_{1} x_{1} \\
\dot{x}_{2} & =[b-\hat{b}(t)] x_{1}-k_{2} x_{2} \\
\dot{x}_{3} & =-[c-\hat{c}(t)] x_{3}-k_{3} x_{3} \\
\dot{x}_{4} & =-[p-\hat{p}(t)] x_{2}-k_{4} x_{4}
\end{aligned}\right.
$$

The parameter estimation errors are defined as

$$
\left\{\begin{array}{l}
e_{a}(t)=a-\hat{a}(t) \\
e_{b}(t)=b-\hat{b}(t) \\
e_{c}(t)=c-\hat{c}(t) \\
e_{p}(t)=p-\hat{p}(t)
\end{array}\right.
$$

In view of (28), we can simplify the plant dynamics (27) as

$$
\left\{\begin{array}{l}
\dot{x}_{1}=e_{a}\left(x_{2}-x_{1}\right)-k_{1} x_{1} \\
\dot{x}_{2}=e_{b} x_{1}-k_{2} x_{2} \\
\dot{x}_{3}=-e_{c} x_{3}-k_{3} x_{3} \\
\dot{x}_{4}=-e_{p} x_{2}-k_{4} x_{4}
\end{array}\right.
$$

Differentiating (28) with respect to $t$, we obtain

$$
\left\{\begin{array}{l}
\dot{e}_{a}(t)=-\dot{\hat{a}}(t) \\
\dot{e}_{b}(t)=-\dot{\hat{b}}(t) \\
\dot{e}_{c}(t)=-\dot{\hat{c}}(t) \\
\dot{e}_{p}(t)=-\dot{\hat{p}}(t)
\end{array}\right.
$$


We use adaptive control theory to find an update law for the parameter estimates.

We consider the quadratic candidate Lyapunov function defined by

$$
V\left(\boldsymbol{x}, e_{a}, e_{b}, e_{c}, e_{p}\right)=\frac{1}{2} \sum_{i=1}^{4} x_{i}^{2}+\frac{1}{2}\left(e_{a}^{2}+e_{b}^{2}+e_{c}^{2}+e_{p}^{2}\right)
$$

Differentiating $V$ along the trajectories of (29) and (30), we obtain

$$
\begin{aligned}
\dot{V}= & -k_{1} x_{1}^{2}-k_{2} x_{2}^{2}-k_{3} x_{3}^{2}-k_{4} x_{4}^{2}+e_{a}\left[x_{1}\left(x_{2}-x_{1}\right)-\dot{\hat{a}}\right] \\
& +e_{b}\left[x_{1} x_{2}-\dot{\hat{b}}\right]+e_{c}\left[-x_{3}^{2}-\dot{\hat{c}}\right]+e_{p}\left[-x_{2} x_{4}-\dot{\hat{p}}\right]
\end{aligned}
$$

In view of (32), we take the parameter update law as

$$
\left\{\begin{array}{l}
\dot{\hat{a}}(t)=x_{1}\left(x_{2}-x_{1}\right) \\
\dot{\hat{b}}(t)=x_{1} x_{2} \\
\dot{\hat{c}}(t)=-x_{3}^{2} \\
\dot{\hat{p}}(t)=-x_{2} x_{4}
\end{array}\right.
$$

Next, we state and prove the main result of this section.

Theorem 1 The novel 4-D hyperchaotic system (25) with unknown system parameters is globally and exponentially stabilized for all initial conditions by the adaptive control law (26) and the parameter update law (33), where $k_{1}, k_{2}, k_{3}, k_{4}$ are positive gain constants.

Proof We prove this result by applying Lyapunov stability theory [75].

We consider the quadratic Lyapunov function defined by (31), which is clearly a positive definite function on $\mathfrak{R}^{8}$.

By substituting the parameter update law (33) into (32), we obtain the time-derivative of $V$ as

$$
\dot{V}=-k_{1} x_{1}^{2}-k_{2} x_{2}^{2}-k_{3} x_{3}^{2}-k_{4} x_{4}^{2}
$$

From (34), it is clear that $\dot{V}$ is a negative semi-definite function on $\mathfrak{R}^{8}$.

Thus, we can conclude that the state vector $\boldsymbol{x}(t)$ and the parameter estimation error are globally bounded, i.e.

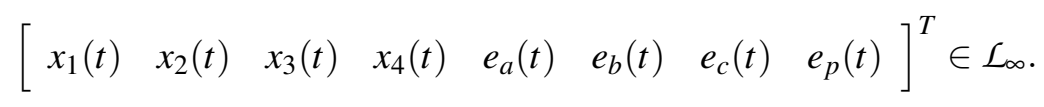

We define $k=\min \left\{k_{1}, k_{2}, k_{3}, k_{4}\right\}$.

Then it follows from (34) that

$$
\dot{V} \leqslant-k\|\boldsymbol{x}(t)\|^{2}
$$


Thus, we have

$$
k\|\boldsymbol{x}(t)\|^{2} \leqslant-\dot{V}
$$

Integrating the inequality (36) from 0 to $t$, we get

$$
k \int_{0}^{t}\|\boldsymbol{x}(\tau)\|^{2} d \tau \leqslant V(0)-V(t)
$$

From (37), it follows that $x \in \mathcal{L}_{2}$.

Using (29), we can conclude that $\dot{x} \in \mathcal{L}_{\infty}$.

Using Barbalat's lemma [75], we conclude that $x(t) \rightarrow 0$ exponentially as $t \rightarrow \infty$ for all initial conditions $\boldsymbol{x}(0) \in \mathfrak{R}^{4}$.

This completes the proof.

For the numerical simulations, the classical fourth-order Runge-Kutta method with step size $h=10^{-8}$ is used to solve the systems (25) and (33), when the adaptive control law (26) is applied.

The parameter values of the novel 4-D hyperchaotic system (25) are taken as in the hyperchaotic case, viz.

$$
a=24, b=125, c=5, p=10
$$

We take the positive gain constants as

$$
k_{1}=5, k_{2}=5, k_{3}=5, k_{4}=5
$$

As initial conditions of the novel 4-D hyperchaotic system (25), we take

$$
x_{1}(0)=12.7, x_{2}(0)=3.8, x_{3}(0)=9.5, x_{4}(0)=6.2
$$

Also, as initial conditions of the parameter estimates, we take

$$
\hat{a}(0)=1.5, \hat{b}(0)=4.9, \hat{c}(0)=2.7, \hat{p}(0)=5.4
$$

In Fig. 5, the exponential convergence of the controlled states of the novel 4-D hyperchaotic system (25) is depicted.

\section{Adaptive synchronization of the novel hyperchaotic systems with unknown parameters}

In this section, we use adaptive control method to derive an adaptive feedback control law for globally synchronizing identical novel 4-D hyperchaotic systems with unknown parameters. 


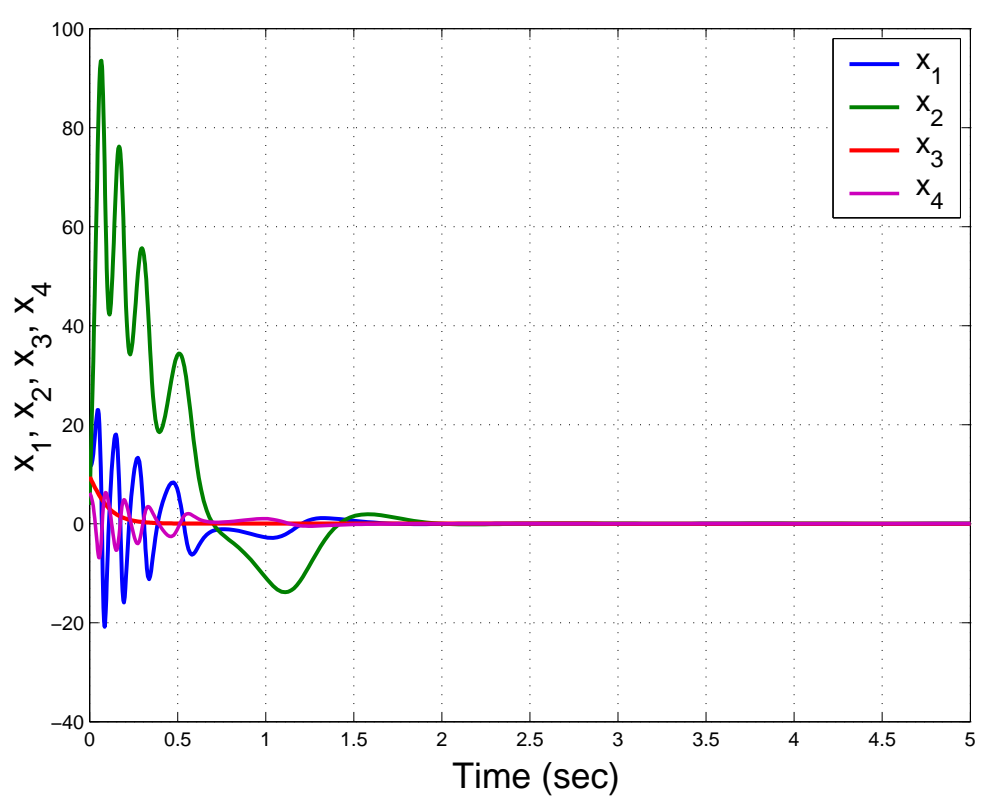

Figure 5: Time-history of the controlled states $x_{1}(t), x_{2}(t), x_{3}(t), x_{4}(t)$

As the master system, we consider the novel 4-D hyperchaotic system given by

$$
\left\{\begin{array}{l}
\dot{x}_{1}=a\left(x_{2}-x_{1}\right)+x_{4} \\
\dot{x}_{2}=b x_{1}-x_{2}-x_{1} x_{3} \\
\dot{x}_{3}=-x_{1}-c x_{3}+x_{1} x_{2}+x_{4} \\
\dot{x}_{4}=-p x_{2}
\end{array}\right.
$$

In (42), $x_{1}, x_{2}, x_{3}, x_{4}$ are the states and $a, b, c, p$ are unknown system parameters. As the slave system, we consider the novel 4-D hyperchaotic system given by

$$
\left\{\begin{array}{l}
\dot{y}_{1}=a\left(y_{2}-y_{1}\right)+y_{4}+u_{1} \\
\dot{y}_{2}=b y_{1}-y_{2}-y_{1} y_{3}+u_{2} \\
\dot{y}_{3}=-y_{1}-c y_{3}+y_{1} y_{2}+y_{4}+u_{3} \\
\dot{y}_{4}=-p y_{2}+u_{4}
\end{array}\right.
$$

In (43), $y_{1}, y_{2}, y_{3}, y_{4}$ are the states and $u_{1}, u_{2}, u_{3}, u_{4}$ are the adaptive controls to be determined using estimates $\hat{a}(t), \hat{b}(t), \hat{c}(t), \hat{p}(t)$ for the unknown parameters $a, b, c, p$, respectively. 
The synchronization error between the novel 4-D hyperchaotic systems (42) and (43) is defined by

$$
\left\{\begin{array}{l}
e_{1}=y_{1}-x_{1} \\
e_{2}=y_{2}-x_{2} \\
e_{3}=y_{3}-x_{3} \\
e_{4}=y_{4}-x_{4}
\end{array}\right.
$$

Then the synchronization error dynamics is obtained as

$$
\left\{\begin{array}{l}
\dot{e}_{1}=a\left(e_{2}-e_{1}\right)+e_{4}+u_{1} \\
\dot{e}_{2}=b e_{1}-e_{2}-y_{1} y_{3}+x_{1} x_{3}+u_{2} \\
\dot{e}_{3}=-e_{1}-c e_{3}+e_{4}+y_{1} y_{2}-x_{1} x_{2}+u_{3} \\
\dot{e}_{4}=-p e_{2}+u_{4}
\end{array}\right.
$$

We consider the adaptive feedback control law

$$
\left\{\begin{array}{l}
u_{1}=-\hat{a}(t)\left(e_{2}-e_{1}\right)-e_{4}-k_{1} e_{1} \\
u_{2}=-\hat{b}(t) e_{1}+e_{2}+y_{1} y_{3}-x_{1} x_{3}-k_{2} e_{2} \\
u_{3}=e_{1}+\hat{c}(t) e_{3}-e_{4}-y_{1} y_{2}+x_{1} x_{2}-k_{3} e_{3} \\
u_{4}=\hat{p}(t) e_{2}-k_{4} e_{4}
\end{array}\right.
$$

where $k_{1}, k_{2}, k_{3}, k_{4}$ are positive gain constants.

Substituting (26) into (45), we get the closed-loop error dynamics as

$$
\left\{\begin{array}{l}
\dot{e}_{1}=[a-\hat{a}(t)]\left(e_{2}-e_{1}\right)-k_{1} e_{1} \\
\dot{e}_{2}=[b-\hat{b}(t)] e_{1}-k_{2} e_{2} \\
\dot{e}_{3}=-[c-\hat{c}(t)] e_{3}-k_{3} e_{3} \\
\dot{e}_{4}=-[p-\hat{p}(t)] e_{2}-k_{4} e_{4}
\end{array}\right.
$$

The parameter estimation errors are defined as

$$
\left\{\begin{array}{l}
e_{a}(t)=a-\hat{a}(t) \\
e_{b}(t)=b-\hat{b}(t) \\
e_{c}(t)=c-\hat{c}(t) \\
e_{p}(t)=p-\hat{p}(t)
\end{array}\right.
$$


In view of (48), we can simplify the plant dynamics (47) as

$$
\left\{\begin{array}{l}
\dot{e}_{1}=e_{a}\left(e_{2}-e_{1}\right)-k_{1} e_{1} \\
\dot{e}_{2}=e_{b} e_{1}-k_{2} e_{2} \\
\dot{e}_{3}=-e_{c} e_{3}-k_{3} e_{3} \\
\dot{e}_{4}=-e_{p} e_{2}-k_{4} e_{4}
\end{array}\right.
$$

Differentiating (48) with respect to $t$, we obtain

$$
\left\{\begin{array}{l}
\dot{e}_{a}(t)=-\dot{\hat{a}}(t) \\
\dot{e}_{b}(t)=-\dot{\hat{b}}(t) \\
\dot{e}_{c}(t)=-\dot{\hat{c}}(t) \\
\dot{e}_{p}(t)=-\dot{\hat{p}}(t)
\end{array}\right.
$$

We use adaptive control theory to find an update law for the parameter estimates. We consider the quadratic candidate Lyapunov function defined by

$$
V\left(\boldsymbol{e}, e_{a}, e_{b}, e_{c}, e_{p}\right)=\frac{1}{2} \sum_{i=1}^{4} e_{i}^{2}+\frac{1}{2}\left(e_{a}^{2}+e_{b}^{2}+e_{c}^{2}+e_{p}^{2}\right)
$$

Differentiating $V$ along the trajectories of (49) and (50), we obtain

$$
\begin{aligned}
\dot{V}= & -k_{1} e_{1}^{2}-k_{2} e_{2}^{2}-k_{3} e_{3}^{2}-k_{4} e_{4}^{2}+e_{a}\left[e_{1}\left(e_{2}-e_{1}\right)-\dot{\hat{a}}\right] \\
& +e_{b}\left[e_{1} e_{2}-\dot{\hat{b}}\right]+e_{c}\left[-e_{3}^{2}-\dot{\hat{c}}\right]+e_{p}\left[-e_{2} e_{4}-\dot{\hat{p}}\right]
\end{aligned}
$$

In view of (52), we take the parameter update law as

$$
\left\{\begin{array}{l}
\dot{\hat{a}}(t)=e_{1}\left(e_{2}-e_{1}\right) \\
\dot{\hat{b}}(t)=e_{1} e_{2} \\
\dot{\hat{c}}(t)=-e_{3}^{2} \\
\dot{\hat{p}}(t)=-e_{2} e_{4}
\end{array}\right.
$$

Next, we state and prove the main result of this section.

Theorem 2 The novel 4-D hyperchaotic systems (42) and (43) with unknown system parameters are globally and exponentially synchronized for all initial conditions by the adaptive control law (46) and the parameter update law (53), where $k_{1}, k_{2}, k_{3}, k_{4}$ are positive gain constants. 
Proof We prove this result by applying Lyapunov stability theory [75].

We consider the quadratic Lyapunov function defined by (51), which is clearly a positive definite function on $\mathfrak{R}^{8}$.

By substituting the parameter update law (53) into (52), we obtain the time-derivative of $V$ as

$$
\dot{V}=-k_{1} e_{1}^{2}-k_{2} e_{2}^{2}-k_{3} e_{3}^{2}-k_{4} e_{4}^{2}
$$

From (54), it is clear that $\dot{V}$ is a negative semi-definite function on $\Re^{8}$.

Thus, we can conclude that the error vector $\boldsymbol{e}(t)$ and the parameter estimation error are globally bounded, i.e.

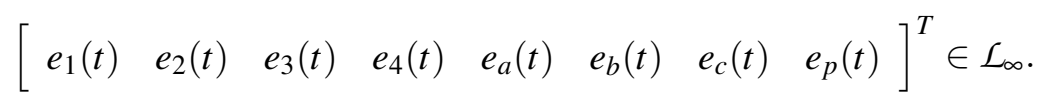

We define $k=\min \left\{k_{1}, k_{2}, k_{3}, k_{4}\right\}$.

Then it follows from (54) that

$$
\dot{V} \leqslant-k\|\boldsymbol{e}(t)\|^{2}
$$

Thus, we have

$$
k\|\boldsymbol{e}(t)\|^{2} \leqslant-\dot{V}
$$

Integrating the inequality (56) from 0 to $t$, we get

$$
k \int_{0}^{t}\|\boldsymbol{e}(\tau)\|^{2} d \tau \leqslant V(0)-V(t)
$$

From (57), it follows that $e \in \mathcal{L}_{2}$.

Using (49), we can conclude that $\dot{e} \in \mathcal{L}_{\infty}$.

Using Barbalat's lemma [75], we conclude that $\boldsymbol{e}(t) \rightarrow 0$ exponentially as $t \rightarrow \infty$ for all initial conditions $\boldsymbol{e}(0) \in \mathfrak{R}^{4}$.

This completes the proof.

For the numerical simulations, the classical fourth-order Runge-Kutta method with step size $h=10^{-8}$ is used to solve the systems (42), (43) and (53), when the adaptive control law (46) is applied.

The parameter values of the novel 4-D hyperchaotic systems are taken as in the hyperchaotic case, viz.

$$
a=24, b=125, c=5, p=10
$$

We take the positive gain constants as $k_{i}=5$ for $i=1, \ldots, 4$.

Furthermore, as initial conditions of the master system (42), we take

$$
x_{1}(0)=5.1, x_{2}(0)=-3.8, x_{3}(0)=4.8, x_{4}(0)=7.6
$$


As initial conditions of the slave system (43), we take

$$
y_{1}(0)=-7.4, \quad y_{2}(0)=13.5, \quad y_{3}(0)=9.2, \quad y_{4}(0)=-2.3
$$

Also, as initial conditions of the parameter estimates, we take

$$
\hat{a}(0)=10.1, \hat{b}(0)=22.4, \hat{c}(0)=14.7, \hat{p}(0)=12.8
$$

Figs. 6-9 describe the complete synchronization of the 4-D novel hyperchaotic systems (42) and (43), while Fig. 10 describes the time-history of the synchronization errors $e_{1}, e_{2}, e_{3}, e_{4}$.

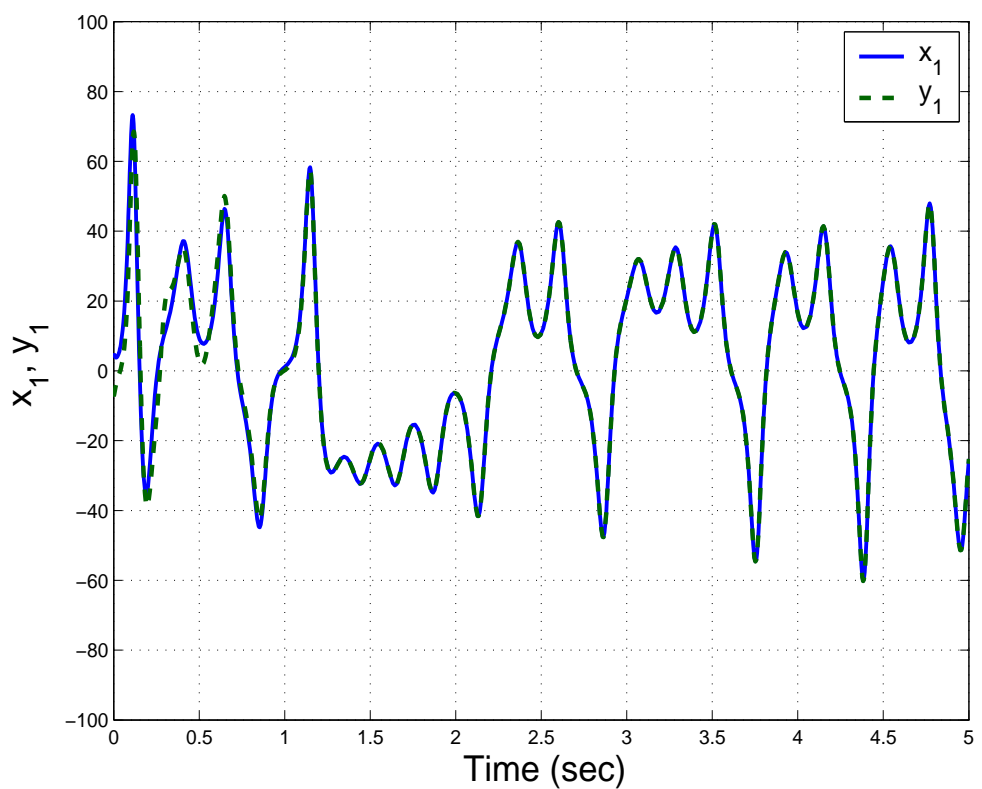

Figure 6: Synchronization of the states $x_{1}$ and $y_{1}$ of the novel hyperchaotic systems

\section{Conclusion}

In this research work, we described an eleven-term novel 4-D hyperchaotic system with two quadratic nonlinearities. We described the qualitative properties of the novel 4$\mathrm{D}$ hyperchaotic system and depicted their phase portraits. We pointed out that the novel 4-D hyperchaotic system has a two-wing attractor. We showed that the novel 4-D hyperchaotic system has two unstable equilibrium points. We calculated the Lyapunov exponents and Kaplan-Yorke dimension of the novel hyperchaotic system. Next,we derived new results for the adaptive control and synchronization of the novel hyperchaotic system with unknown parameters. MATLAB simulations have been shown to demonstrate all the main results derived in this research work. 


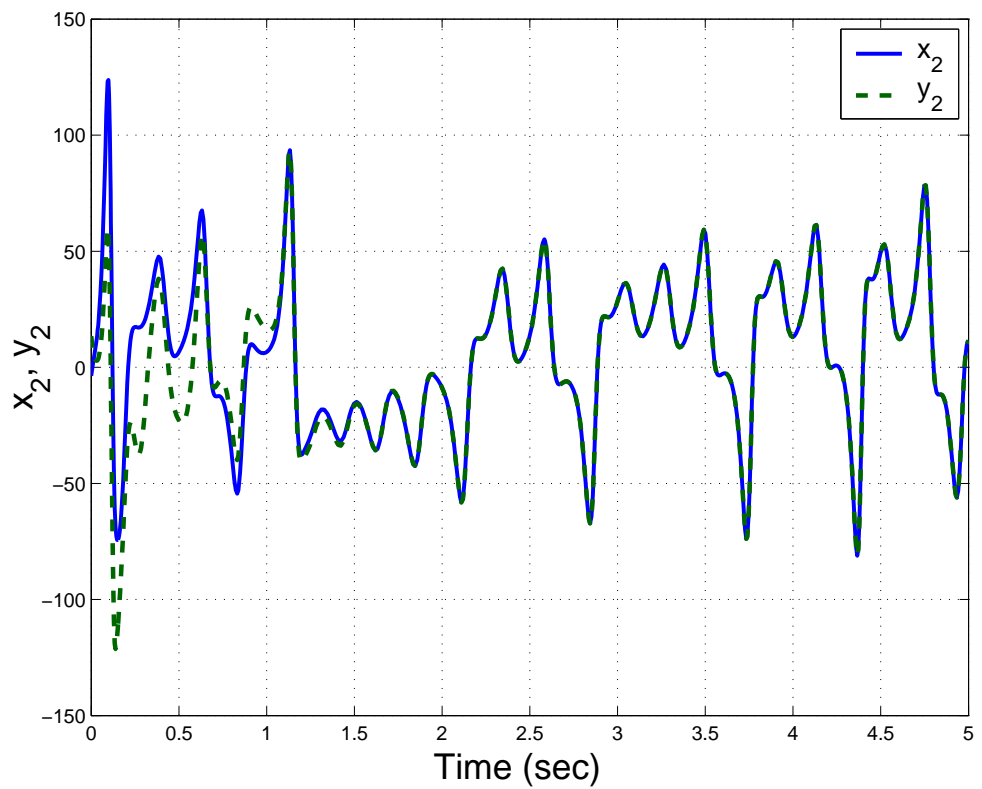

Figure 7: Synchronization of the states $x_{2}$ and $y_{2}$ of the novel hyperchaotic systems

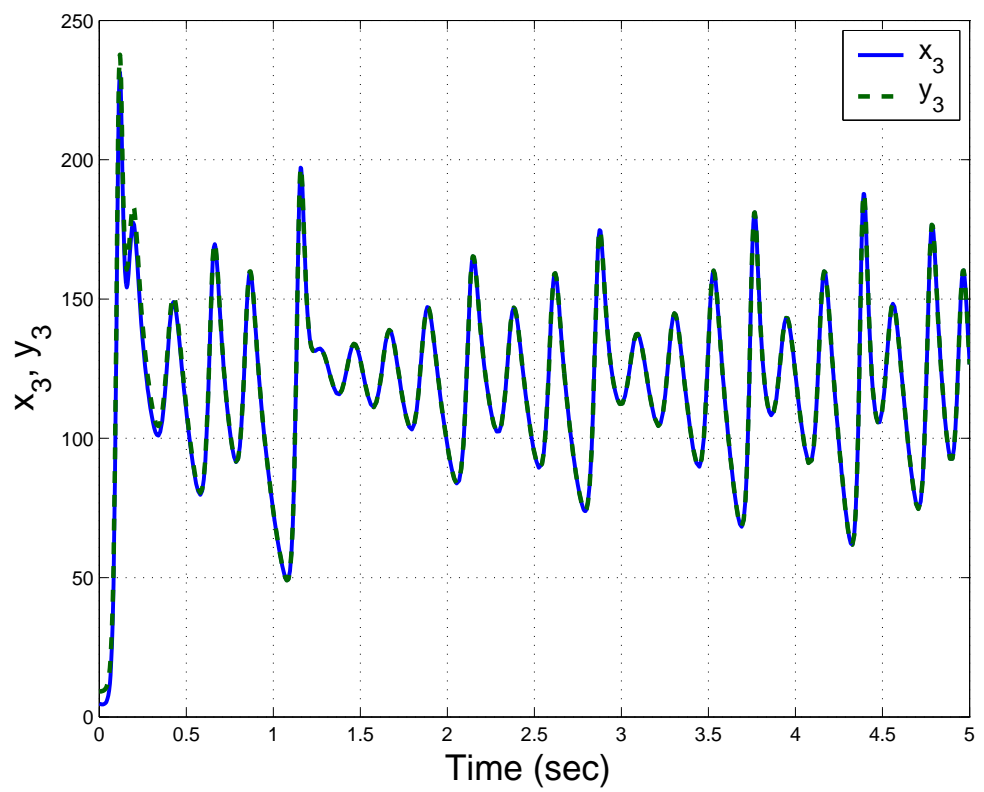

Figure 8: Synchronization of the states $x_{3}$ and $y_{3}$ of the novel hyperchaotic systems 


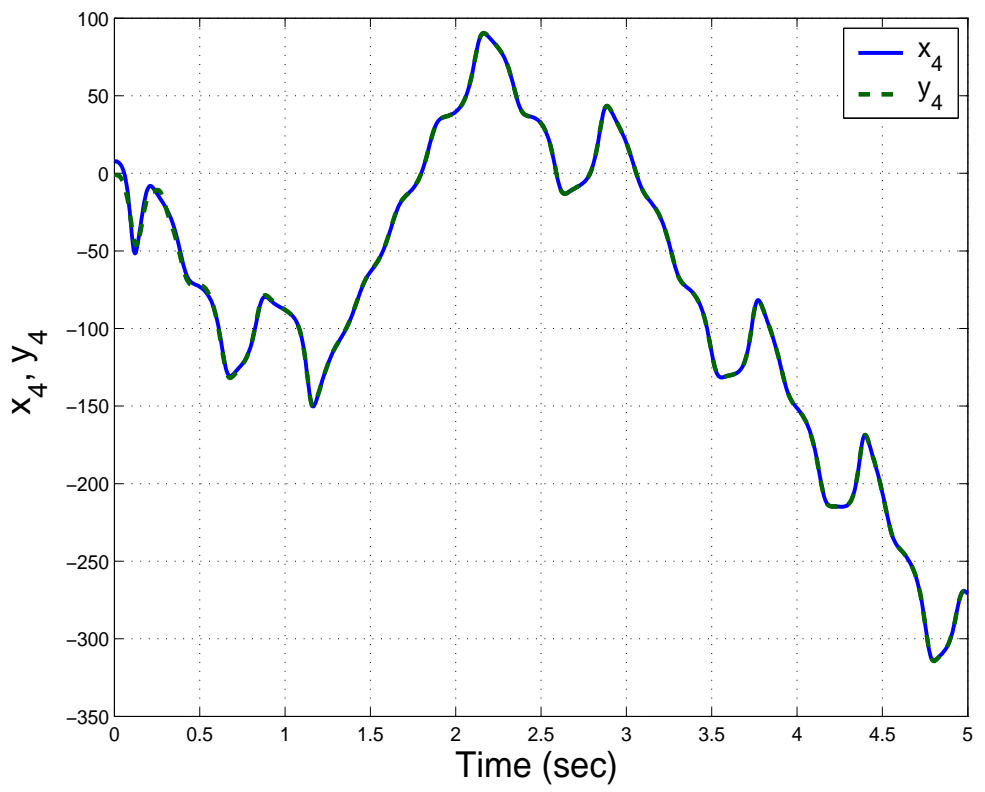

Figure 9: Synchronization of the states $x_{4}$ and $y_{4}$ of the novel hyperchaotic systems

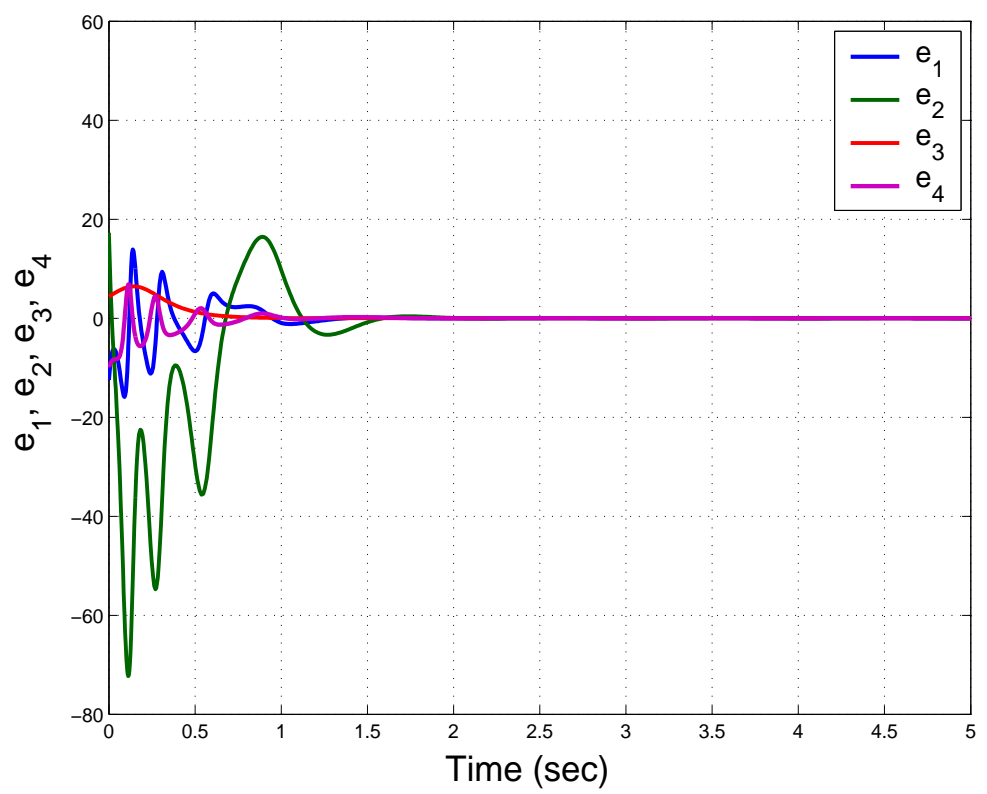

Figure 10: Time-history of the synchronization errors $e_{1}, e_{2}, e_{3}, e_{4}, e_{5}$ 


\section{References}

[1] S. VAIDYANATHAN and C. VOLOS: Advances and Applications in Chaotic Systems. Berlin, Springer-Verlag, 2016.

[2] S. VAIDYANATHAN and C. VOLOS: Advances and Applications in Nonlinear Control Systems. Berlin, Springer-Verlag, 2016.

[3] D. RUELLE and F. TAKENS: On the nature of turbulence. Communications in Mathematical Physics, 20 (1971), 167-192.

[4] R.M. MAY: Limit cycles in predator-prey communities. Science, 177 (1972), 900908.

[5] M.J. FEIGENBAUM: Universal behaviour in nonlinear systems. Physica D: Nonlinear Phenomena, 7 (1983), 16-39.

[6] E.N. LORENZ: Deterministic nonperiodic flow. Journal of the Atmospheric Sciences, 20 (1963), 130-141.

[7] O.E. RÖSSLER: An equation for continuous chaos. Physics Letters A, 57 (1976), 397-398.

[8] A. ARNEODO, P. COULLET and C. TRESSER: Possible new strange attractors with spiral structure. Communications in Mathematical Physics, 79 (1981), 573579 .

[9] J.C. SPROTT: Some simple chaotic flows. Physical Review E, 50 (1994), 647-650.

[10] G. CHEN and T. UETA: Yet another chaotic attractor. International Journal of Bifurcation and Chaos, 9 (1999), 1465-1466.

[11] J. LÜ and G. CHEN: A new chaotic attractor coined. International Journal of Bifurcation and Chaos, 12 (2002), 659-661.

[12] C.X. LIU, T. LIU, L. LIU and K. LIU: A new chaotic attractor. Chaos, Solitons and Fractals, 22 (2004), 1031-1038.

[13] G. CAI and Z. TAN: Chaos synchronization of a new chaotic system via nonlinear control. Journal of Uncertain Systems, 1 (2007), 235-240.

[14] G. TIGAN and D. OPRIS: Analysis of a 3D chaotic system. Chaos, Solitons and Fractals, 36 (2008), 1315-1319.

[15] D. LI: A three-scroll chaotic attractor. Physics Letters A, 372 (2008), 387-393.

[16] V. SUNDARAPANDIAN and I. PEHLIVAN: Analysis, control, synchronization and circuit design of a novel chaotic system. Mathematical and Computer Modelling, 55 (2012), 1904-1915. 
[17] V. SUNDARAPANDIAN: Analysis and anti-synchronization of a novel chaotic system via active and adaptive controllers. Journal of Engineering Science and Technology Review, 6 (2013), 45-52.

[18] S. VAIDYANATHAN: A new six-term 3-D chaotic system with an exponential nonlinearity. Far East Journal of Mathematical Sciences, 79 (2013), 135-143.

[19] S. VAIDYANATHAN: Analysis and adaptive synchronization of two novel chaotic systems with hyperbolic sinusoidal and cosinusoidal nonlinearity and unknown parameters. Journal of Engineering Science and Technology Review, 6 (2013), 53-65.

[20] S. VAIDYANATHAN: A new eight-term 3-D polynomial chaotic system with three quadratic nonlinearities. Far East Journal of Mathematical Sciences, 84 (2014), 219-226.

[21] S. VAIDYANATHAN: Analysis, control and synchronisation of a six-term novel chaotic system with three quadratic nonlinearities. International Journal of Modelling, Identification and Control, 22 (2014), 41-53.

[22] S. VAIDYANATHAN and K. MADHAVAN: Analysis, adaptive control and synchronization of a seven-term novel 3-D chaotic system. International Journal of Control Theory and Applications, 6 (2013), 121-137.

[23] S. VAIDYANATHAN: Analysis and adaptive synchronization of eight-term 3-D polynomial chaotic systems with three quadratic nonlinearities. European Physical Journal: Special Topics, 223 (2014), 1519-1529.

[24] S. VAIDYANATHAN, C. VOLOS, V.T. PHAM, K. MADHAVAN and B.A. IDOWU: Adaptive backstepping control, synchronization and circuit simulation of a 3-D novel jerk chaotic system with two hyperbolic sinusoidal nonlinearities. Archives of Control Sciences, 24 (2014), 257-285.

[25] I. PEHLIVAN, I.M. MOROZ and S. VAIDYANATHAN: Analysis, synchronization and circuit design of a novel butterfly attractor. Journal of Sound and Vibration, 333 (2014), 5077-5096.

[26] A. AKGUL, I. MOROZ, I. PEHLIVAN and S. VAIDYANATHAN: A new fourscroll chaotic attractor and its engineering applications. Optik, 127 (2016), 5491Ü5499.

[27] S. JAFARI and J.C. SPROTT: Simple chaotic flows with a line equilibrium. Chaos, Solitons and Fractals, 57 (2013), 79-84.

[28] V.T. PHAM, S. JAFARI, C. VOLOS, S. VAIDYANATHAN and T. KAPITANIAK: A chaotic system with infinite equilibria located on a piecewise linear curve. Optik, 127 (2016), 9111-9117. 
[29] V.T. PHAM, S. VAIDYANATHAN, C. VOLOS, S. JAFARI and S.T. KINGNI: A no-equilibrium hyperchaotic system with a cubic nonlinear term. Optik, 127 (2016), 3259-3265.

[30] V.T. PHAM, C. VOLOS, S. JAFARI, S. VAIDYANATHAN, T. KAPITANIAK and X. WANG: A chaotic system with different families of hidden attractors. International Journal of Bifurcation and Chaos, 26 (8), (2016), 1650139.

[31] V.T. PHAM, S. JAFARI, C. VOLOS, A. GIAKOUMIS, S. VAIDYANATHAN and T. KAPITANIAK: A chaotic system with equilibria located on the rounded square loop and its circuit implementation. IEEE Transactions on Circuits and Systems-II: Express Briefs, 63 (9), (2016), 878-882.

[32] O.I. TACHA, C.K. VOLOS, I.M. KYPRIANIDIS, I.N. STOUBOULOS, S. VAIDYANATHAN and V.T. PHAM: Analysis, adaptive control and circuit simulation of a novel nonlinear finance system. Applied Mathematics and Computation, 276 (2016), 200-217.

[33] V.T. PHAM, C.K. VOLOS and S. VAIDYANATHAN: Multi-scroll chaotic oscillator based on a first-order delay differential equation. Studies in Computational Intelligence, 581 (2015), 59-72.

[34] S. VAIDYANATHAN: Output regulation of the forced Van der Pol chaotic oscillator via adaptive control method. International Journal of PharmTech Research, 8 (6), (2015), 106-116.

[35] P. GASPARD: Microscopic chaos and chemical reactions. Physica A: Statistical Mechanics and its Applications, 263 (1999), 315-328.

[36] Q.S. LI and R. ZHU: Chaos to periodicity and periodicity to chaos by periodic perturbations in the Belousov-Zhabotinsky reaction. Chaos, Solitons \& Fractals, 19 (2004), 195-201.

[37] M. KYRIAZIS: Applications of chaos theory to the molecular biology of aging. Experimental Gerontology, 26 (1991), 569-572.

[38] E. CARLEN, R. CHATELIN, P. DEGOND and B. WENNBERG: Kinetic hierarchy and propagation of chaos in biological swarm models. Physica D: Nonlinear Phenomena, 260 (2013), 90-111.

[39] I. SUÁREZ: Mastering chaos in ecology. Ecological Modelling, 117 (1999), 305314.

[40] J.C. SPROTT, J.A. VANO, J.C. WILDENBERG, M.B. ANDERSON and J.K. NOEL: Coexistence and chaos in complex ecologies. Physics Letters A, 335 (2005), 207-212. 
[41] S. VAIDYANATHAN: Global chaos control of 3-cells cellular neural network attractor via integral sliding mode control. International Journal of PharmTech Research, 8 (8), (2015), 211-221.

[42] S. VAIDYANATHAN: Global chaos synchronization of 3-cells cellular neural network attractors via integral sliding mode control. International Journal of PharmTech Research, 8 (8), (2015), 118-130.

[43] S. VAIDYANATHAN: Global chaos regulation of a symmetric nonlinear gyro system via integral sliding mode control. International Journal of ChemTech Research, 9 (5), (2016), 462-469.

[44] S. VAIDYANATHAN: Synchronization of Tokamak systems with symmetric and magnetically confined plasma via adaptive control. International Journal of ChemTech Research, 8 (6), (2015), 818-827.

[45] S. VAIDYANATHAN: Dynamics and control of Tokamak system with symmetric and magnetically confined plasma. International Journal of ChemTech Research, $\mathbf{8}$ (6), (2015), 795-803.

[46] C.K. VOLOS, D. PROUSALIS, I.M. KYPRIANIDIS, I. STOUBOULOS, S. VAIDYANATHAN and V.T. PHAM: Synchronization and anti-synchronization of coupled Hindmarsh-Rose neuron models. International Journal of Control Theory and Applications, 9 (1), (2016), 101-114.

[47] S. VAIDYANATHAN: Global chaos control of the FitzHugh-Nagumo chaotic neuron model via integral sliding mode control. International Journal of PharmTech Research, 9 (4), (2016), 413-425.

[48] S. VAIDYANATHAN: Adaptive synchronization of the identical FitzHughNagumo chaotic neuron models. International Journal of PharmTech Research, 8 (6), (2015), 167-177.

[49] S. VAIDYANATHAN, C.K. VOLOS, K. RAJAGOPAL, I.M. KYPRIANIDIS and I.N. STOUBOULOS: Adaptive backstepping controller design for the antisynchronization of identical WINDMI chaotic systems with unknown parameters and its SPICE implementation. Journal of Engineering Science and Technology Review, 8 (2), (2015), 74-82.

[50] C.K. VOLOS, V.T. PHAM, S. VAIDYANATHAN, I.M. KYPRIANIDIS and I.N. STOUBOULOS: Synchronization phenomena in coupled Colpitts circuits. Journal of Engineering Science and Technology Review, 8 (2), (2015), 142-151.

[51] J.C. SPROTT: Elegant Chaos. Singapore, World Scientific, 2010. 
[52] C. LI, X. LIAO and K.W. WONG: Lag synchronization of hyperchaos with application to secure communications. Chaos, Solitons and Fractals, 23 (2005), 183193.

[53] N. SMAOUI, A. KAROUMA and M. ZRIBI: Secure communications based on the synchronization of the hyperchaotic Chen and the unified chaotic systems. Communications in Nonlinear Science and Numerical Simulation, 16 (2011), 3279-3293.

[54] X.J. WU, H. WANG and H.T. LU: Hyperchaotic secure communication via generalized function projective synchronization. Nonlinear Analysis: Real World Applications, 12 (2011), 1288-1299.

[55] T.I. CHIEN and T.L. LIAO: Design of secure digital communication systems using chaotic modulation, cryptography and chaotic synchronization. Chaos, Solitons \& Fractals, 24 (2005), 241-245.

[56] B. ZHANG, M. CHEN and D. ZHOU: Chaotic secure communication based on particle filtering. Chaos, Solitons \& Fractals, 30 (2006), 1273-1280.

[57] X. WU, C. BAI and H. KAN: A new color image cryptosystem via hyperchaos synchronization. Communications in Nonlinear Science and Numerical Simulation, 19 (2014), 1884-1897.

[58] Q. ZHANG, L. GUO and X. WEI: A novel image fusion encryption algorithm based on DNA sequence operation and hyper-chaotic system. Optik - International Journal for Light and Electron Optics, 124 (2013), 3596-3600.

[59] G. YE and J. ZHOU: A block chaotic image encryption scheme based on selfadaptive modelling. Applied Soft Computing, 22 (2014), 351-357.

[60] H. LIU, X. WANG and A. KADIR: Color image encryption using Choquet fuzzy integral and hyper chaotic system. Optik - International Journal for Light and Electron Optics, 124 (2013), 3257-3533.

[61] A. BUSCARINO, L. FORTUNA and M. FRASCA: Experimental robust synchronization of hyperchaotic circuits. Physica D: Nonlinear Phenomena, 238 (2009), 1917-1922.

[62] N. YUJUN, W. XINGYUAN, W. MINGJUN and Z. HUAGUANG: A new hyperchaotic system and its circuit implementation. Communications in Nonlinear Science and Numerical Simulation, 15 (2010), 3518-3524.

[63] X. WEI, F. YUNFEI and L. QIANG: A novel four-wing hyper-chaotic system and its circuit implementation. Procedia Engineering, 29 (2012), 1264-1269.

[64] P. ZHOU and K. HUANG: A new 4-D non-equilibrium fractional-order chaotic system and its circuit implementation. Communications in Nonlinear Science and Numerical Simulation, 19 (2014), 2005-2011. 
[65] O.E. RÖSSLER: An equation for hyperchaos. Physics Letters A, 71 (1979), 155157.

[66] Q. JIA: Hyperchaos generated from the Lorenz chaotic system and its control. Physics Letters A, 366 (2007), 217-222.

[67] A. CHEN, J. LU, J.LÜ and S. YU: Generating hyperchaotic Lü attractor via state feedback control. Physica A, 364 (2006), 103-110.

[68] X. LI: Modified projective synchronization of a new hyperchaotic system via nonlinear control. Communications in Theoretical Physics, 52 (2009), 274-278.

[69] J. WANG and Z. CHEN: A novel hyperchaotic system and its complex dynamics. International Journal of Bifurcation and Chaos, 18 (2008), 3309-3324.

[70] D. GHOSH and S. BHATTACHARYA: Projective synchronization of new hyperchaotic system with fully unknown parameters. Nonlinear Dynamics, 61 (2010), $11-21$.

[71] S. VAIDYANATHAN, C. VOLOS, V.T. PHAM and K. MADHAVAN: Analysis, adaptive control and synchronization of a novel 4-D hyperchaotic hyperjerk system and its SPICE implementation. Archives of Control Sciences, 25 (1), (2015), 135158.

[72] S. VAIDYANATHAN: Analysis, adaptive control and synchronization of a novel 4-D hyperchaotic hyperjerk system via backstepping control method. Archives of Control Sciences, 26 (3), (2016), 311-318.

[73] S. VAIDYANATHAN: Adaptive controller and synchronizer design for the QiChen chaotic system. Lecture Notes of the Institute for Computer Sciences, SocialInformatics and Telecommunications Engineering, 85 (2012), 124-133.

[74] S. VAIDYANATHAN and S. RASAPPAN: Hybrid synchronization of hyperchaotic Qi and Lü systems by nonlinear control. Communications in Computer and Information Science, 131 (2011), 585-593.

[75] H.K. KHALIL: Nonlinear Systems. Prentice Hall, New Jersey, 1996. 